\title{
Surfaces and interfaces: combining electronic structure and electron transport models for describing electron spectra
}

\author{
László Kövér* \\ Institute for Nuclear Research, Hungarian Academy of Sciences, Debrecen, Hungary
}

Keywords: surfaces, interfaces, electronic structure, electron transport, electron spectra, models/simulations

\section{Scales of Physical Phenomena Reflected in Electron Spectra Induced from Atoms, Molecules, Nanostructures, and Solids by Photons or Charged Particles}

There are many different types of physical and chemical phenomena reflected in electron spectra induced from atoms, molecules, nanostructures, and solids by photons or charged particles. Table 1 compares the spatial, time, and typical excitation energy scales of selected phenomena.

OPEN ACCESS

Edited by:

Simone Taioli,

Bruno Kessler Foundation, Italy;

Charles University,

Czech Republic

Reviewed by:

Lucrezia Aversa,

National Research Council, Italy

Lucia Calliari,

Bruno Kessler Foundation, Italy

*Correspondence:

László Kövér

Ikover@atomki.mta.hu

Specialty section:

This article was submitted to

Mechanics of Materials, a section of the journal Frontiers in Materials

Received: 27 January 2015 Accepted: 02 April 2015

Published: 22 April 2015

Citation:

Kövér L (2015) Surfaces and interfaces: combining electronic structure and electron transport models for describing electron

Front. Mater. 2:35

doi: 10.3389/fmats.2015.00035
The data (Krausz and Ivanov, 2009; Surdutovich and Solov'yov, 2014) in Table 1 demonstrate that the phenomena influencing electron spectra take place on very wide scales, sometimes encompassing differences of many orders of magnitude. This suggests the need for multiscale approaches when extracting electronic structure or chemical analytical information from electron spectra.

\section{Electron Spectra}

Electron spectra induced from solids contain information on (i) the electronic structure (local at atomic, molecular, cluster, and surface/interface level - or delocalized in the bulk of the solid), different single or multiple excitations, and the chemical state resolved chemical composition; (ii) the (ordered, disordered, or amorphous) physical structure of the solid, as well as the lateral or indepth concentration distribution of the constituent chemical species, via effects of electron transport phenomena (including electron diffraction). While core and valence band photoelectron as well as Auger electron spectra reflect the structure of occupied electronic states, shake-up satellites, due to excitations localized at atomic or molecular level, reflect the structure of unoccupied electronic levels. In the case of shake-off excitations, the energy is continuously shared between the two emitted photoelectrons; therefore, the contribution of these phenomena cannot be separated in the photoelectron spectra from the continuous energy distribution background of electrons scattered inelastically in the solid. Shake-off excitations, however, result in energy loss satellite peaks in photoinduced Auger spectra.

Core photoelectron peaks carry localized signatures characteristic of the atomic environment of the emitting atom, while valence band photoelectron spectra reflect properties of delocalized electronic states. On the other hand, information on the site projected density of valence electron states can be obtained from the analysis of core-core valence (CCV) or CVV Auger spectra. The shape of the core photoelectron lines can, however, be influenced by delocalized excitations (e.g., by excitation of electron-hole pairs in the conduction bands of metals or by "intrinsic" type plasmon excitations due to the appearance of the core hole). 


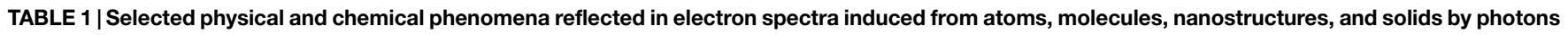
or charged particles: spatial and time scales, typical excitation energies (Krausz and Ivanov, 2009; Surdutovich and Solov'yov, 2014).

\begin{tabular}{|c|c|c|c|}
\hline Phenomena & $\begin{array}{c}\text { Spatial } \\
\text { scale }(\mathrm{nm})\end{array}$ & Time scale (s) & $\begin{array}{c}\text { Typical excitation } \\
\text { energies (examples, eV) }\end{array}$ \\
\hline Atomic/molecular primary ionization & $0.1-10$ & $10^{-18}-10^{-14}$ & $\begin{array}{c}\gg 10 \text { (atomic cores) } \\
\gg 1 \text { (valence levels in molecules) }\end{array}$ \\
\hline Chemical reactions & 1 & $10^{-12}-10^{-5}$ & \\
\hline $\begin{array}{l}\text { Excitation processes } \\
\text { (a) In free atoms, molecules, nanostructures } \\
\text { (b) In atoms and molecules embedded in solids }\end{array}$ & $\begin{array}{c}10^{-3}-1 \\
10^{-2}-10\end{array}$ & $\begin{array}{l}10^{-18}-10^{-15} \\
10^{-15}-10^{-12}\end{array}$ & \\
\hline $\begin{array}{l}\text { Some photon induced processes in solids } \\
\text { (a) Plasmon excitation due to sudden creation of core holes } \\
\text { (b) Coster-Kronig Auger transitions, electron-hole pair exc. in conduction bands of metals } \\
\text { (c) Transition between vibration levels of atoms in molecules in solids } \\
\text { (d) Phonon excitation (in photoemission) } \\
\text { (e) Transport of secondaries }\end{array}$ & $0.1-10$ & $\begin{array}{c}10^{-17}-10^{-16} \\
10^{-15}-10^{-14} \\
10^{-13} \\
10^{-18}-10^{-14}\end{array}$ & $\begin{array}{c}\sim 1-10 \\
0.1-1 \\
\sim 10^{-3} \\
0.1\end{array}$ \\
\hline
\end{tabular}

From the point of view of energy loss of photoexcited electrons emitted from solids, "intrinsic" type excitations, connected with the suddenly created core holes localized on the core ionized atoms, usually lead to energy loss satellite structures in the electron spectra. Moreover, all electrons emitted from solids are affected by excitations occurring at surface crossings ("surface excitations," localized in two dimensions) and resulting in energy losses, leading to surface plasmon satellite peaks. Finally, collective excitations of weakly bound, free, or near free electrons in the solid by photoinduced electrons during their transport within the solid ("extrinsic" excitations) lead to delocalized volume excitations manifested, e.g., in the presence of bulk plasmon satellite peaks in the spectra. It should be noted that interferences can take place between surface and bulk, extrinsic, and intrinsic collective excitation phenomena.

\section{Modeling/Interpreting Electron Spectra Using Classical and Quantum Frame Models}

For describing or simulating the electronic structure and effects of electron transport on electron spectra emitted from solids, combinations of models based on different approximations are used. Here, only some examples are mentioned for illustration. "One step” quantum mechanical (QM) models include full QM calculations accounting for all phenomena, while the "two- or morestep" models assume that different phenomena can be separated. A recent report (Minár et al., 2011) on "one step" models describing angle resolved photoelectron spectra provides examples of applications to metals and alloys.

An example of "one step" QM models is the quantum Landau theory (QLT) developed by Fujikawa et al. (2008). This model explains all plasmon energy loss features occurring due to core level photoemission from solids and fully accounts for intrinsic and extrinsic plasmon excitations and interferences between these processes, for multiple plasmon energy loss features in the spectra, as well as for effects of multiple elastic electron scatterings (including photoelectron diffraction) preceding and following the energy loss of photoelectrons. For high kinetic energy photoelectrons, the QLT performs well, for $\sim 100 \mathrm{eV}$ kinetic energy photoelectrons the model was successfully applied in the case of $\mathrm{Al} 2 \mathrm{~s}, \mathrm{Na} 2 \mathrm{~s}$, and $\mathrm{Li}$ 1s spectra photoexcited from single crystals (Kazama et al., 2014; Niki et al., 2014).

While "one step" QM models require demanding calculations even in the case of simple ordered systems and it is difficult to include multiple electron scattering, "two- or more-step" models usually assume the separability of the intrinsic and extrinsic processes and use semiclassical or classical approximations for describing the latter phenomena, neglecting interference effects between intrinsic and extrinsic or surface and bulk processes in most cases. This practically means the separation of electron transport and photoionization/excitation processes, focusing on near surface electron transport.

For describing effects of electron transport in solids, different approaches can be followed. A possibility is to deconvolute contributions of electron transport from measured electron spectra in order to obtain spectra carrying information on the (local) electronic structure. An example is the Monte Carlo (MC) simulation-based deconvolution technique for removing contributions due to different kinds (intrinsic, extrinsic, surface, bulk) of multiple plasmon excitations from X-ray photoelectron spectra. A method based on the partial intensity analysis (PIA) approximation (Werner, 2001) using a linearized Boltzmann-type kinetic equation accounts for multiple elastic electron scattering as well, while neglecting core hole and interference effects. The contribution from intrinsic plasmon excitations is assumed to be similar to that from the bulk extrinsic excitations and removed by applying an "adaptive deconvolution." This approach seems to work especially well, e.g., in the case of high energy photoelectron and Auger spectra excited from 3d metals. In this particular case, the asymmetry of the core photoelectron lineshapes (due to the sudden creation of electron-hole pairs in the conduction band upon core level photoionization) can be described using an independent model (Hughes and Scarfe, 1996) based on the joint density of electronic states (that can be obtained from density functional calculations) around the atom with the core hole (Novák et al., in preparation). The MC method based on the parameters of the PIA model, combined with an expert system containing extended databases on physical data characterizing photoionization and electron transport in solids, is applicable 
for simulation of X-ray photoelectron and Auger spectra excited from solid structures (Werner et al., 2014). It should be noted, however, that accurate simulation of photoelectron spectra is a very demanding task due to the very complex assembly of physical phenomena to be accounted for.

A different approach is first to model/simulate "intrinsic" spectra on the basis of the available knowledge on the atomic and electronic structure, then to simulate the contribution of electron transport to "intrinsic" spectra with the aim to directly compare the resulted synthetic spectra to experimental electron spectra. An example of such an approach is using a mixed QM and MC method for describing Auger spectra photoexcited from $\mathrm{SiO}_{2}$ nanoclusters of different sizes (Taioli et al., 2009a,b). Within this approach first the "intrinsic" Auger spectra were obtained from cluster $a b$ initio QM calculations then the effects of inelastic energy losses of Auger electrons within the nanoclusters on the Auger lineshapes were simulated using the MC method. Synthesized and measured spectra compare fairly well (Taioli et al., 2009a,b). The model assumes fixed nuclei (so phonons are not considered). However, shake-up and shake-off transitions, though neglected in Taioli et al. (2009b), are foreseen by the model.

An example of models describing excitations due to the core hole and to photoelectrons during their transport in solids is the semiclassical dielectric response model (DRM) (Tougaard and Yubero, 2004), which includes the polarization accompanying photoelectrons on their way toward the surface within the solid, near the surface inside and outside of the solid as well as the influence of the stationary hole during the whole photoemission process. The primary excitation spectrum and the dielectric function are input physical quantities to the DRM that, in addition to bulk excitations, describes core hole effects (intrinsic excitations), surface excitations as well as interference effects (between intrinsic and extrinsic or surface and bulk excitations) (Tougaard and Yubero, 2004). On the other hand, if one has detailed knowledge (e.g., from independent experiments or models) on the parametrized primary excitation spectrum, the actual parameter values (energy position, energy widths, and intensity of the component peaks/structures) can be derived from the measured photoelectron spectrum with the help of the DRM and the measured photoelectron spectrum. First, the effective cross section for inelastic electron scattering can be derived using the DRM, then this cross section is convoluted with the parametrized primary excitation spectrum varying the parameters until achieving a good

\section{References}

Fujikawa, T., Kazama, M., and Shinotsuka, H. (2008). Theoretical study of plasmon losses in core-level photoemission spectra. e-J. Surf. Sci. Nanotech. 6, 263-268. doi:10.1380/ejssnt.2008.263

Hughes, H. P., and Scarfe, J. A. (1996). Lineshapes in core-level photoemission from metals 1 . Theory and computational analysis. J. Phys. Condens. Matter 8, 1421-1438.

Kazama, M., Shinotsuka, H., Ohori, Y., Niki, K., Fujikawa, T., and Kövér, L. (2014). Plasmon losses in core-level photoemission spectra studied by the quantum Landau formula including full multiple scattering. Phys. Rev. B 89, 045110. doi:10.1103/PhysRevB.89.045110

Kövér, L. (2010). X-ray photoelectron spectroscopy using hard X-rays. J. Electron Spectrosc. Relat. Phenomena 178-179, 241-257. doi:10.1016/j.elspec.2009. 12.004 agreement with the experimental XPS spectrum (Pauly et al., 2014). A recent study applying the DRM for determination of the $\mathrm{Cu} 2 \mathrm{p}$ primary excitation spectrum in $\mathrm{CuO}$ demonstrates (Pauly et al., 2014) the use of this approach yielding results agreeing with those predicted by the charge transfer multiplet model calculations. The DRM, however, while accounting for multiple inelastic electron scattering, neglects the effects of multiple elastic electron scattering.

\section{Challenges in Characterization of Advanced Materials: Need for Multiscale Materials Analysis and Modeling}

Development of advanced materials, e.g., layered structure doped nanocomposites requests 3D monitoring of their chemical, physical, and electronic structure on multiple scales as a function of their mechanical, optical, and electric properties. Such a need for multiscale materials analysis, however, poses great challenges for the available methods of characterization and usually multimethod approaches are needed.

The increasingly popular, non-destructive HArd X-ray PhotoElectron Spectroscopy (HAXPES) with its flexible applicability for chemical state resolved studies of surface (at grazing photon incidence) and deeply (several $10 \mathrm{~nm}$ ) buried interface structures, is one of the promising candidate methods for multiscale analysis (Kövér, 2010). The information depth of HAXPES using grazing photon incidence (at total X-ray reflection conditions) is set by the depth of penetration of X-rays and it is limited to the uppermost atomic layers. On the contrary, tuning the photon energy in the case of near normal photon incidence makes the deeply buried interfaces accessible. Angular resolved HAXPES and HAXPES using X-ray standing waves for excitation combined with advanced modeling allow accurate quantitative chemical analysis of concentration depth profiles of deep regions.

\section{Summary}

Electron spectra of solids reflect phenomena on different spatial and time scales and events at various levels of localization. Combining models/simulations for local electronic structure and electron transport is necessary for accurate interpretation of experimental electron spectra. Analysis of advanced materials requires novel methods and models for multiscale approaches.

Krausz, F., and Ivanov, M. (2009). Attosecond physics. Rev. Mod. Phys. 81, 163-234. doi:10.1103/RevModPhys.81.163

Minár, J., Braun, J., Mankovsky, M., and Ebert, H. (2011). Calculation of angleresolved photo emission spectra within the one-step model of photo emission recent developments. J. Electron Spectrosc. Relat. Phenomena 184, 91-99. doi:10. 1016/j.elspec.2011.01.009

Niki, K., Yamamura, N., Ohori, N. Y., Kazama, M., Fujikawa, T., and Kövér, L. (2014). Theoretical study of plasmon losses from Li 1s level in core-level photoemission spectra. Surf. Interface Anal. 46, 924-926. doi:10.1002/sia.5504

Pauly, N., Tougaard, S., and Yubero, F. (2014). Determination of the Cu 2p primary excitation spectra for $\mathrm{Cu}, \mathrm{Cu} 2 \mathrm{O}$ and CuO. Surf. Sci. 620, 17-22. doi:10.1016/j. susc.2013.10.009

Surdutovich, E., and Solov'yov, A. V. (2014). Multiscale physics of ion-induced radiation damage. Appl. Radiat. Isot. 83, 100-104. doi:10.1016/j.apradiso.2013. 01.035 
Taioli, S., Simonucci, S., and Dapor, M. (2009a). SURPRISES: when ab initio meets statistics in extended systems. Comput. Sci. Disc. 2, 015002. doi:10.1088/ 1749-4699/2/1/015002

Taioli, S., Simonucci, S., Calliari, L., Filippi, M., and Dapor, M. (2009b). Mixed ab initio quantum mechanical and Monte Carlo calculations of secondary emission from SiO2 nanoclusters. Phys. Rev. B 79, 085432. doi:10.1103/PhysRevB.79. 085432

Tougaard, S., and Yubero, F. (2004). QUEELS software package for calculation of surface effects in electron spectra. Surf. Interface Anal. 36, 824-827. doi:10.1002/ sia. 1774

Werner, W. S. M. (2001). Electron transport in solids for quantitative surface analysis. Surf. Interface Anal. 31, 141-176. doi:10.1002/sia.973

Werner, W. S. M., Smekal, W., and Powell, C. J. (2014). NIST Database for the Simulation of Electron Spectra for Surface Analysis, Ver. 2.0, NIST
Standard Reference Database 100. Available at: http://www.nist.gov/srd/ nist100.cfm

Conflict of Interest Statement: The author declares that the research was conducted in the absence of any commercial or financial relationships that could be construed as a potential conflict of interest.

Copyright (c) 2015 Kövér. This is an open-access article distributed under the terms of the Creative Commons Attribution License (CC BY). The use, distribution or reproduction in other forums is permitted, provided the original author(s) or licensor are credited and that the original publication in this journal is cited, in accordance with accepted academic practice. No use, distribution or reproduction is permitted which does not comply with these terms. 